\title{
Automated Multidimensional Characterization of Power Amplifier for Design and Production
}

\author{
Charles Nader ${ }^{1,2}$ \\ ${ }^{1}$ Center for RF Measurement Technology \\ University of Gävle \\ Gävle, SE-801 76, Sweden \\ ${ }^{2}$ Signal Processing Lab, \\ Royal Institute of Technology \\ Stockholm, SE-100 44, Sweden \\ Charles.nader@hig.se
}

\author{
Hibah Altahir ${ }^{1}$, Olav Andersen ${ }^{3}$, Niclas Björsell ${ }^{1}$, \\ Edith Condo $^{1}$, Niclas Keskitalo ${ }^{1,4}$, Hector de la Rosa \\ ${ }^{3}$ Ericsson $\mathrm{AB}$ \\ Gävle, SE-800 06, Sweden \\ ${ }^{4}$ Ericsson $\mathrm{AB}$ \\ Stockholm, SE-164 80, Sweden \\ ${ }^{5}$ Freescale Semiconductor Nordic AB \\ Kista, SE-164 26, Sweden
}

\begin{abstract}
Designing, optimizing and producing modern power amplifiers (PA) requires new and fast RF (radio frequency) measurement techniques capable of characterizing its real behavior. PAs are a truly multidimensional device where many desired performance parameters are contradictory to each other. This is especially true for the generation of modern communication PAs that require high efficiency, high linearity as well as high bandwidth. This paper presents a software-defined measurement setup for fast and cost efficient multidimensional measurements based on highly accurate standard instruments and a PC. The test bed as well as the graphical user interface is presented along with a demonstration of its functionality. During tuning of tank networks, drain quiescent current, and bias conditions, 3-dimensional graphs can be selected for the most appropriate axes of trade-off parameters to display a true behavior of the PA under test subjected to real-world or close to real-world signals. The measurement system offers the possibility to monitor envelope-tracking dynamic power consumption up to $100 \mathrm{MHz}$ plus the possibility to use high crest factors.
\end{abstract}

Keywords-Automated multidimensional measurements, power amplifier, time domain measurement, state-of-the-art test-bed, gaindistortion-efficiency

\section{INTRODUCTION}

Designers strive to develop a power amplifier (PA) (or a system including a PA) with the best properties for the application. It is well known that a PA working with modern communication signals have a large number of tradeoffs between different parameters. As the demands on high performance PAs continue to grow, novel measurement algorithms are needed as enabler for efficient design, testing, and characterization of future power devices. A multidimensional representation of the PA's figure of merits is a crucial tool for tuning in on the best performance tradeoffs and selecting the optimum operating point; that is the drain quiescent current, $\mathrm{I}_{\mathrm{DQ}}$, the frequency range and the power range near compression. Modern PAs dealing with multiple carrier signals at high bandwidth, high crest-factors as well as high linearity are truly multidimensional devices. Traditionally, these performance parameters are presented in tables and twodimensional plots. Optimizing the performance in the multidimensional device based on this two-dimensional information is both difficult and time consuming. Thus, multidimensional characterizations offer unique possibilities to make tradeoffs in several dimensions, i.e. superior design and production advantages.

To our knowledge, only $[1,2]$ have considered this need from the test engineers and designers point of view. In addition, [3, 4] characterize multidimensional models of PAs for linearization by using pre-distortion techniques. The models techniques given in $[3,4]$ are used for existing PAs and not aimed for designing, tuning and production test of PAs.

In this paper we suggest a software defined measurement (SDM) method to measure and present critical parameters such as distortion, gain, and efficiency in three-dimensional plots in order to offer a design view in several dimension and multidimensional tuning in production. In fact, presenting the above three merits in one single plot will allow designers to optimize the operating point of the PA based on the best suitable combination for the application. The measurement set up offers large possibilities compared to traditional methods regarding speed and accuracy.

\section{MEASUREMENT SYSTEM}

Major challenges in designing an automated test system are accuracy, speed and cost. Thus, the used instruments are highly accurate standard instruments, without any special designed equipment. All instruments are connected to a computer that controls the measurements, process the measured data and presents the result. That is, a softwaredefined measurement setup.

\section{A. Test-bed}

The test-bed is based on a vector signal generator (VSG), R\&S SMU200A, a vector signal analyzer (VSA), R\&S FSQ26, an oscilloscope, Agilent 54610B, a current probe, Agilent N2783A, a highly linear driver amplifier, Ericsson 3G PA, a controllable power supply, Agilent E3631A and a personal computer (PC), see Figure 1. The instruments are connected to the PC via LAN and/or via GPIB interface.

This work was supported by Ericsson AB, Freescale Semiconductor Nordic AB, Infineon Technologies Nordic AB, Knowledge Foundation, NOTE $\mathrm{AB}$, Rohde \& Schwarz AB and Syntronic AB. 


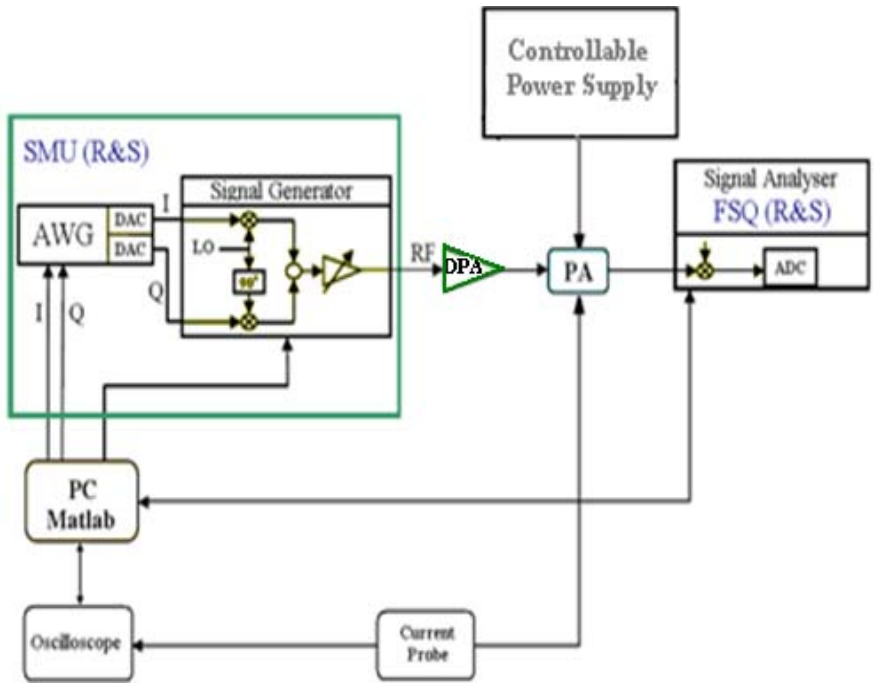

Figure 1. The measurement setup

Such instruments, or similar to, are mainly found in most of RF laboratories which doesn't impose any additional hardware cost.

\section{B. Measurement method}

A baseband signal is generated in the PC. The signal is a consecutive baseband-sequence of test scenarios, where the amplitude and frequency are increased stepwise. By using coherent sampling, it was assured that the aimed signal power in the spectrum is contained within the relative tone respectively [5]. The signal is then downloaded to an arbitrary waveform generator (AWG) in the VSG, where it is upconverted to radio frequencies (RF). The main advantage of using an AWG is that we can perform several test scenarios in one measurement, and thus improve the speed of the characterization. Fig. 2 shows a sequence of 15 power steps for a two-tone signal.

In order to have an accurate and stable power stepping, the total root-mean square (RMS) power of the carrier should be found. A power formula was derived that links the total RMS power of the carrier, $\mathrm{RMS}_{\mathrm{T}}$, the highest RMS power step in channel, $\mathrm{HS}_{\mathrm{RMS}}$, and the crest factor $(\mathrm{CF})$ of the full steps sequence, $\mathrm{CF}_{\mathrm{T}}$. Starting from the general relation between $\mathrm{CF}_{\mathrm{T}}$, $\mathrm{RMS}_{\mathrm{T}}$ and peak power, Peak , in a logarithmic basis, as in

$$
R M S_{T}=P e a k_{T}-C F_{T},
$$

Peak $\mathrm{T}_{\mathrm{T}}$ can be developed to represent $\mathrm{HS}_{\mathrm{RMS}}$ added to the $\mathrm{CF}$ of one single step, $\mathrm{CF}_{\mathrm{S}}$, as in

$$
\operatorname{Peak}_{T}=H S_{R M S}+C F_{S} .
$$

For a multitone system with $\mathrm{N}$ tones, $\mathrm{HS}_{\mathrm{RMS}}$ represents the highest RMS of total tones that should be reached in the sweep. For a Wideband Code Division Multiple Access (WCDMA) system $\mathrm{HS}_{\mathrm{RMS}}$ represents the highest step of total power in channel that should be reached in the sweep.

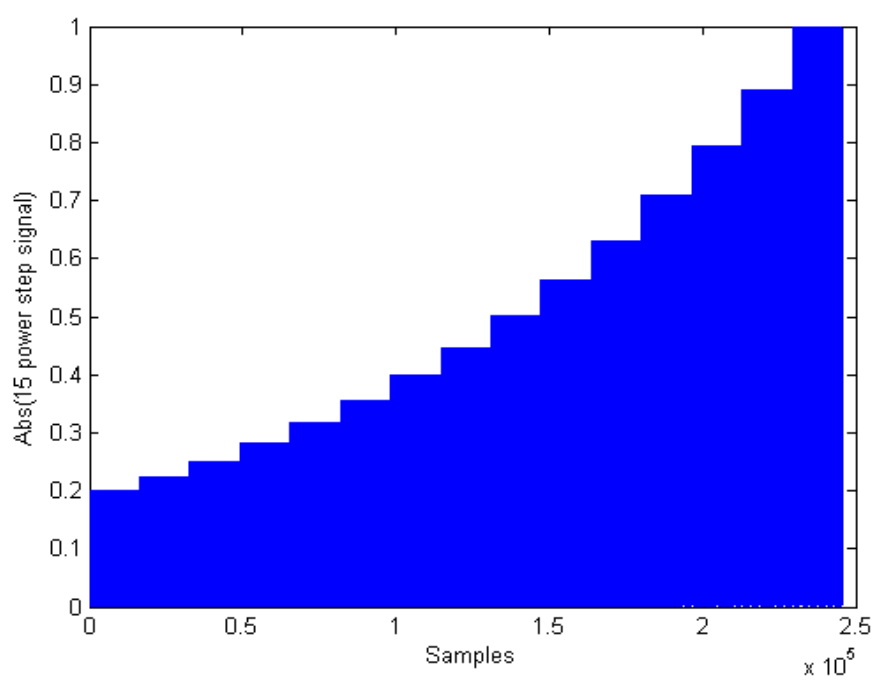

Figure 2. Envelope of a power sweep sequence

To accurately monitor the current vector of the drain supply, a high bandwidth hall sensor current probe is used. Drain supply current to the PA is then measured by an oscilloscope. By this method, envelope-tracking dynamic power consumption can be accurately measured up to about $100 \mathrm{MHz}$. The output power from the PA is measured by the VSA, which can be accurately calibrated by a sensing power meter to obtain $\pm 0.35 \mathrm{~dB}$ accuracy, even for modulated time variant signals.

In order to provide a design tool to select the optimum $\mathrm{I}_{\mathrm{DQ}}$, a controllable power supply is used to sweep the gate bias of the device under test (DUT) at a percentage of $\pm x \%$. A lookup table is used to transfer the bias voltage to the $\mathrm{I}_{\mathrm{DQ}}$. Stepping bias voltage is also used to study the performance of the PA for different classes of operation, identify its sweet spots and their stability with the operating class.

The measured data is sent to the PC, where the data sequences are synchronized in time and frequency domain and each test scenario (amplitude and frequency) is separated and processed individually [5]. A requirement for that is the use of time repetitive signals.

One of the main key points in the measurement system is the synchronization between input and output signals, and also between output signal and relative current measurements. The in-phase (I) and quadrature (Q) data on a Nyquist scale are collected from the VSA. The synchronization is done in two steps. The first step is done in the time domain, where crosscorrelation is used to synchronize data to one-sample accuracy. The second step is done in the frequency domain, where interpolation in the phase information is used to identify the exact data sequence relative to each measurement step.

Synchronizing the current measurements with relative signal sequence is more complex due to difference in sampling frequency between the oscilloscope and the VSG and existence of current glitches in the beginning of a sequence. A decoupler is used on the drain side to eliminate unwanted fluctuations due to sudden short in the power supply. The current trace is collected from the oscilloscope through GPIB. Moreover, a 
fifth order Butterworth low-pass digital filter is used to reduce measurement noise in the data. The oscilloscope floor noise is subtracted from the filtered data and to the result a sign function implemented in the software is applied which gives positive pulses $(+1)$ for positive samples and negative pulses (1 ) for negative ones. The outcome is differentiated in a series form which will result in negative pulses $(-2)$ when the power goes from the highest step to the lowest one and positive pulses $(+2)$ when the power steps up its first level. By choosing any two consecutive negative pulses, the period of current sequence is detected. A demonstration of current synchronization based on slopes identification is presented in Fig. 3.

Another method for current measurements would be to resample the collected data from the oscilloscope in correspondence to the power sequence length, and then apply the same synchronization procedure used for power measurements. The drawback is the loss of speed in the processing due to resampling.

\section{Visualization}

The result is presented in a 3D plot where gain, efficiency and distortion can be the three axes. A graphical user interface (GUI) has been designed in order to show the multidimensional plots, but also for parameter settings, see Fig. 4 and Fig. 5. The settings are the $\mathrm{I}_{\mathrm{DQ}}$, frequency and input power ranges. A marker can be used to get numerical data at a specific point in the plot. The marker presents the three parameters but also the test conditions (power and frequency) for that specific measurement. Moreover, the GUI can also present data in the traditional two-dimensional plots.

To facilitate the possibility to tune the PA properly and select the most suitable quiescent point, a similar to "live update" of the plots is accessible. The GUI has slide bars, where the $\mathrm{I}_{\mathrm{DQ}}$ or the gate voltage $(\mathrm{Vg})$ can be altered and the plots update the performance of the PA simultaneously.

\section{RESULTS}

The system performance for different power levels and frequencies are shown in Fig. 6 and Fig. 7 where dynamic range and power calibration are investigated. Input and output link to the DUT are connected to each other through an adaptor with a gain of $-0.2 \mathrm{~dB}$. Fig. 6 presents the dynamic range of the system (including the driver). An average value of $-60 \mathrm{dBc}$ can be achieved at an input level of $40 \mathrm{dBm}$. The noise created in the system is mainly due to the $20 \mathrm{MHz}$ IQ bandwidth and to the $53 \mathrm{~dB}$ attenuators used in the output link. Fig. 7 shows the variation of the system gain regarding frequency and input power. A peak to peak variation of $0.4 \mathrm{~dB}$ is found up to 45 $\mathrm{dBm}$ in the band of 2.1-2.2 GHz, which is the flat band of the driver amplifier used.

To investigate the measurement system deeper, GUI and the new figure of merits, a class AB LDMOS WCDMA high power $(130 \mathrm{~W})$ high gain amplifier is measured. Fig. 4 shows gain, efficiency versus distortion measured using a two-tone input signal while Fig. 5 shows corresponding results using a WCDMA signal.

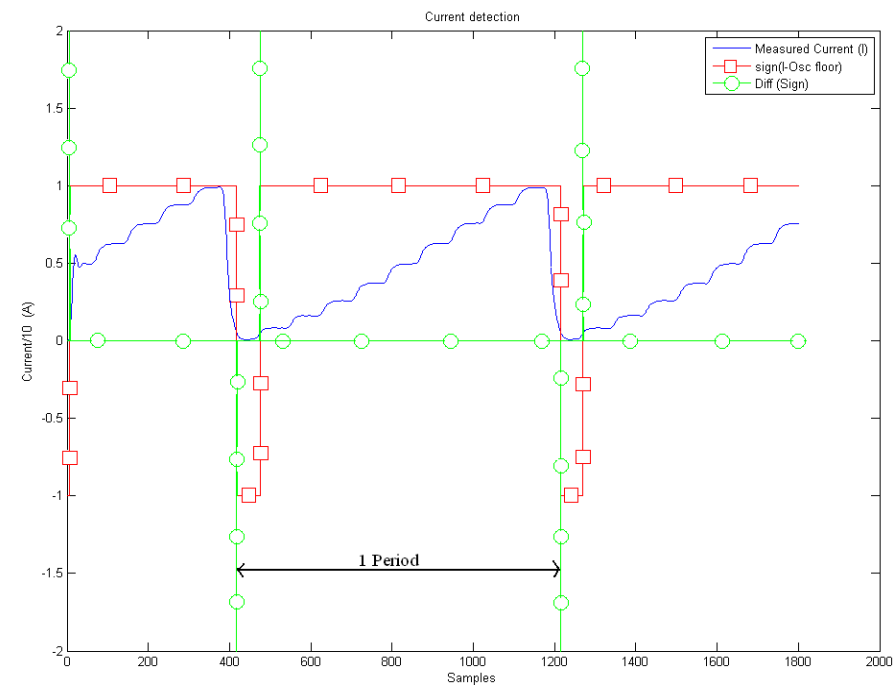

Figure 3. Current Detection and Synchronization based on digital filtering and second order differentiation.

The gain of the PA is the ratio of output power $\left(\mathrm{P}_{\mathrm{OUT}}\right)$ to input power $\left(\mathrm{P}_{\mathrm{IN}}\right)$, and is measured in decibels. The efficiency is the power added efficiency (PAE). The distortion parameter depends on the excitation signal. The distortion considered in the case of the two-tone signals is the third order intermodulation distortion (IM3) whereas adjacent channel power ratio (ACPR) is considered in the case of WCDMA signals [6].

From such representation, designers can choose the operation region of their products with respect to different figure of merits in a fast and reliable way. In addition, investigating the operation of an amplifier based on different bias levels and the variation of the above figure of merits with respect to that will give an insight in the nature of the device and help in characterizing it.

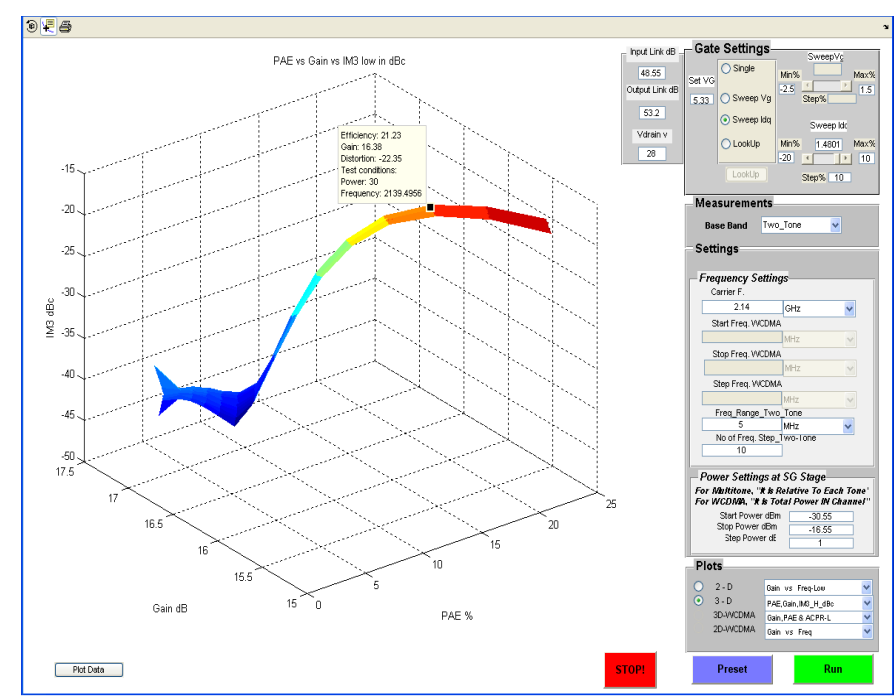

Figure 4. Three-dimensional plot of gain, efficiency and distortion measured on a high power amplifier using a two-tone input signal. 


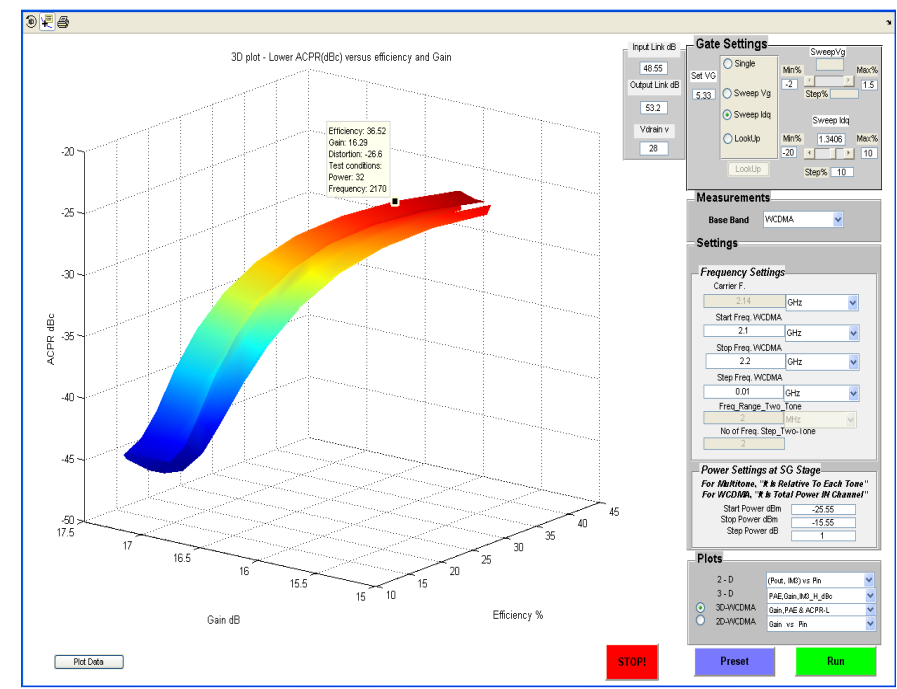

Figure 5. Three-dimensional plot of gain, efficiency and distortion measured on a high power amplifier using a WCDMA input signal.

One important and attractive result a PA designer would like to see is the sweet spot presented as a dip in the IM3 products. Such sweet spots offer an increase of several dB in the carrier to IM3 ratio. A know property is the relation between the existence of those sweet spots, their power level position, and the amplifier class of operation [7]. By changing the $\mathrm{I}_{\mathrm{DQ}}$ current slider, one can identify the existence of such sweet spots and find the suitable operation point relative to it in a similar to "live update" result.

Finally, by investigating the variation of the IM3 with the tone spacing, asymmetry between IM3 channels can be identified and modeled for best linearization performance [8].

\section{DISCUSSION}

The measurement system shows strong malleability regarding tuning factors from bias, power and frequency aspect and covers most of the figure of merits a PA designer would like to investigate. In this first prototype only two types of signals are implemented; 2-tone and WCDMA, but the design is general and the GUI can be extended to include more type of signals.

A high bandwidth hall sensor current probe is used to accurately monitor the current vector of the drain supply. Supply current to the PA is then measured by an oscilloscope; by this method an envelope tracking dynamic power consumption can be accurately measured up to $100 \mathrm{MHz}$. In fact, this property is taken further by investigating the maximization of PAE using bias modulation.

More measurement features can be added to the system in order to improve its performance. Implementing coherent averaging can improve the dynamic range of the receiver up to $20 \mathrm{~dB}$ [5]. Receivers are never ideal, a bandwidth limitation on their baseband can vary up to $20-50 \mathrm{MHz}$; using frequency stitching can allow measurements of signals as wide as hundreds of $\mathrm{MHz}$ [5].

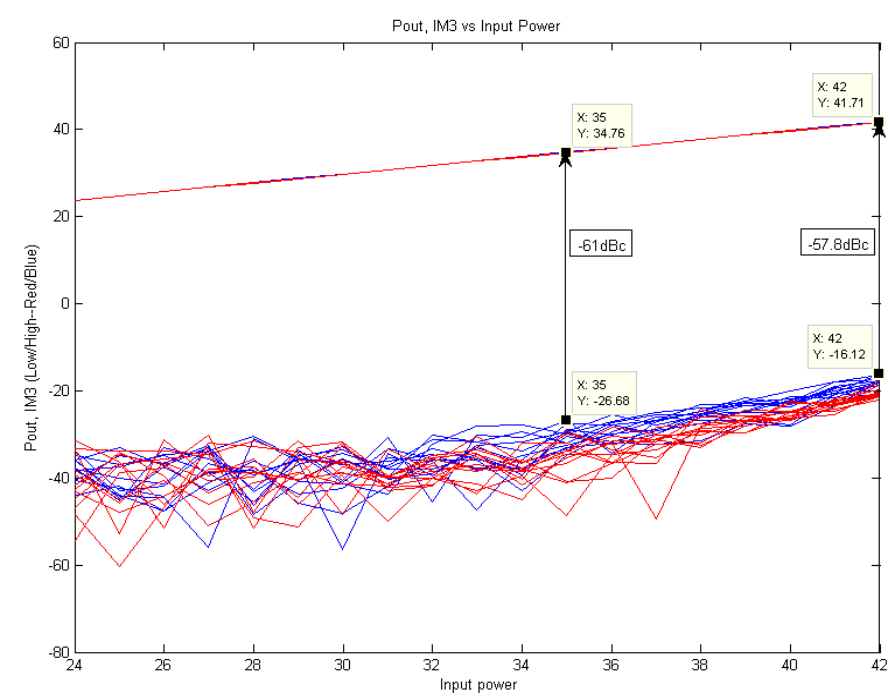

Figure 6. Dynamic range versus input power.

Finally, excitation signal purity is a critical issue in a measurement system; applying digital pre-distortion (DPD) can reduce the spurious in the input link to the DUT [9].

\section{A. Measurement Speed and Accuracy}

Measurement speed and accuracy is a critical issue nowadays as RF measurement engineers compete for the best state-of-the-art system for testing and production. Using stair sequences is a fast method for power sweeping. However some limitations in speed and accuracy have been observed and investigated. In fact the used oscilloscope was the bottleneck in the system due to its slow data transferring protocol and small number of samples in its trace which limited the number of steps that can be swept. To clarify this limitation, time consumption with and without current measurement is presented for sequences formed by 8192 samples/step. The system is capable of running for example 10 frequency steps and 20 power steps in 180 seconds (140 seconds without current measurement). While adding 10 bias steps will results in a 2000 measurements in 30 minutes (23 minutes without current measurement).

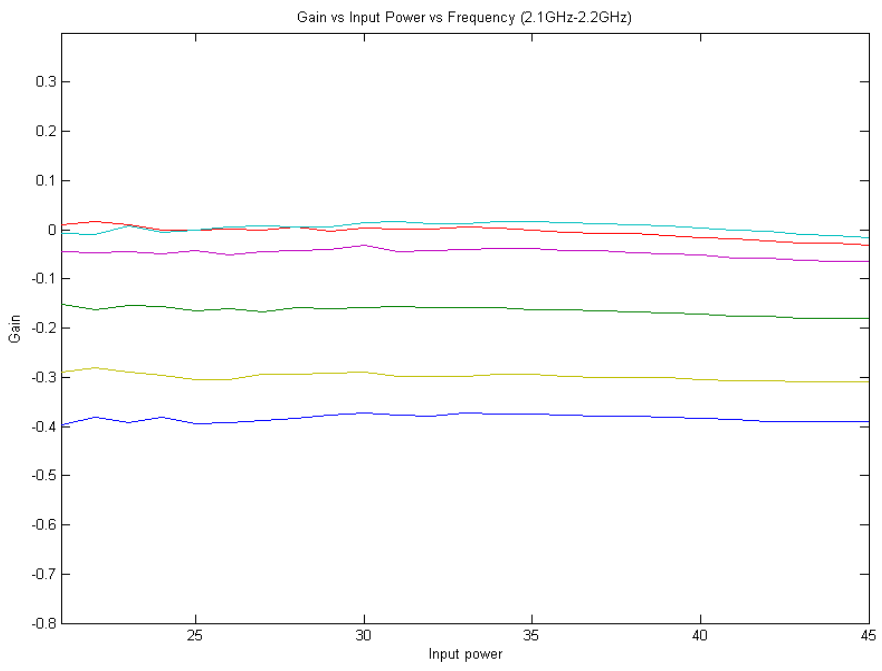

Figure 7. Gain variation versus input power and frequency. 


\section{B. Faster Approach}

A faster method would be to sweep both frequency and power in baseband by using a series stair sequences representing both dimensions. By this method the same number of frequency and power steps is expected to be measured in 3540 seconds (without current measurements it is done in 28 seconds). While adding 10 bias steps and current measurements are expected to be in 6.5 minutes. This new sweeping approach by modulating the baseband in both frequency and power domain will decrease the time consumption in measurements dramatically; however it will put more requirements on equipments and processing, certainly if a large number of steps are required.

In fact using such long sequences (on the order of millions of samples) will include limitations regarding processing data e.g. Fourier transform and cross correlation for synchronization and hardware limitations regarding processors, memories and current measurements through normal oscilloscope. Solutions for that are under investigation.

In fact, measuring the current by the use of an external ADC can easily solve the current measurement limitation. Another method would be to input the current as I or Q baseband input to the analyzer and sweep between RF and baseband input in measuring, while synchronization is similar to the power signal one.

\section{CONCLUSION}

In this paper, a software defined measurement for multidimensional PA characterization and testing is developed and verified. It is an enabler for efficient design and characterization of PAs.

New figure of merit is presented that shows gain and efficiency versus distortion. A lookup table and a slide bar are used to vary the $I_{D Q}$. By this method, an insight study of the PA can be made and the best operation conditions can be determined. Two types of excitation signals are implemented in the GUI; two-tone and WCDMA signal. However the GUI is malleable and can easily be extended to support further type of signals.

A fast approach in measurement is presented. Sweeping both frequency and power in a sequence of series stairs is introduced and evaluated. It shows a dramatic reduction in time consumption of the measurements.

\section{REFERENCES}

[1] T. Driver, "Device Performance Trade-Offs Easily Explored Using New Software and Measurement Methodology," in ARFTG Conference Digest-Spring, 53rd, 1999, pp. 1-9.

[2] Jie Hu, K.G. Gard, N.B Carvalho, and M.B. Steer, "Time-frequency characterization of long-term memory in nonlinear power amplifiers," IEEE MTT-S Int. Microwave Sympo. Dig., Atlanta, GA, 2008, pp. 269272.

[3] R. N. Braithwaite, "A self-generating coefficient list for machine learning in RF power amplifiers using adaptive predistortion," IEEE $36^{\text {th }}$ European Microwave Conference, Manchester, UK, 2006, pp. 12291232 .

[4] J. Goodman, B. Miller, G. Raz, and M. Herman, "A new approach to achieving high-performance power amplifier linearization," IEEE Radar Conference 2007, Boston, MA, USA, 2007, pp. 840-845.

[5] D. Wisell, "Measurement Techniques for Characterization of Power Amplifiers." vol. Doctorial Thesis Stockholm, Sweden: Royal Institute of Technology, 2007.

[6] S. C. Cripps, RF Power Amplifiers for Wireless Communications, Second ed. Norwood, MA: Artech House, 2006.

[7] C. Fager, J.C. Pedro, N. B. de Carvalho, and H. Zirath, "Prediction of IMD in LDMOS Transistor Amplifiers using a New Large-Signal Model", IEEE Transactions on Microwave Theory and Techniques 2002 , vol.50, pp. 2834-2842.

[8] N. Borges de Carvalho and J. C. Pedro, 'Two-tone IMD Asymmetry in Microwave Power Amplifiers," IEEE MTT-S Int. Microwave Sympo. Dig., Boston, MA, 2000, vol.1, pp. 445-448.

[9] C. Luque and N. Björsell, "Improved dynamic range for multi-tone signal using model-based pre-distortion," 13th Workshop on ADC Modeling and Testing, Florence, Italy, 2008, pp. 1003-1007. 